\title{
ANALYTICAL METHOD DEVELOPMENT AND VALIDATION: A REVIEW
}

\section{Mr. Mahesh S Patil, Dr. Rvindra. R. Patil Dr. Shailesh. S. Chalikwar, Dr. Sanjay J Surana, Dr. Sandip D Firke}

Department of Pharmaceutical Quality Assurance. R. C. Patel Institute of Pharmaceutical Education and Research, Shirpur.

Conflicts of Interest: Nil

Corresponding author: Mahesh S Patil

\section{ABSTRACT}

The development of sound Analytical method is of supreme importance during the process of drug discovery, release to market and development, culminating in a marketing approval. The objective of this paper is to review the method development, optimize and validation of the method for the drug product from the developmental stage of the formulation to commercial batch of the product.The purpose of this validation is to show that processes involved in the development and manufacture of drug, production and analytical testing can be performed in an effective and reproducible manner. This review article provides guidance on how to perform validation characteristics for the analytical method which are utilized in pharmaceutical analysis.

Keywords: Analytical method validation, Pharmaceutical analysis, Specificity, Precision, Accuracy.

\subsection{Introduction:}

High Performance Liquid Chromatography (HPLC) is one of the most used analytical techniques. Chromatographic process can be defined as separation technique involving mass-transfer between stationary and mobile phase. HPLC utilizes a liquid mobile phase to separate the components of a mixture. The stationary phase can be a liquid or a solid phase. This component are first dissolved in a solvent, and then forced to flow through a chromatographic column under a high pressure. In the column, the mixture separates into its components. The amount of resolution is important, and is dependent upon the extent of interaction between the solute components and the stationary phase. The stationary phase is defined as the immobile packing material in the column. The interaction of the solute with mobile and stationary phases can be manipulated through different choices of both solvents and stationary phases. As a result, HPLC acquires a high degree of versatility not found in other chromatographic systems and it has the ability to easily separate a wide variety of chemicalmixtures ${ }^{1}$.

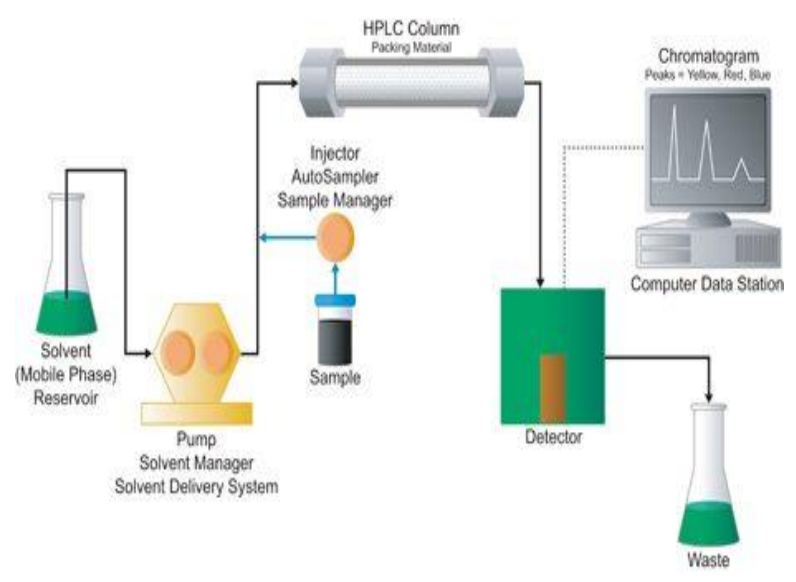

Figure 1: Flow Diagram of HPLC

$>$ High resolution

$>$ Small diameter, Stainless steel, Glass column

$>$ Rapid analysiss

$>$ Relatively higher mobile phase pressure

$>$ Controlled flow rate of mobile phase

\subsection{Analytical Method Development:}

Analytical method development is the process by which a specific analytical method is to be developed for drug products from the stage of in process to finished product and minivalidation to be done before starting the 
analyses of routine samples, investigation samples and stability samples. Analytical method development and finalizing the method consists of

$>$ Standardizing the working standard from reference standard.

> Optimizing the chromatographic condition, concentration of standard and sample solution and extraction procedure of the sample.

$>$ Analytical method verification or mini validation to be done before analyzing (routine samples) tests like assay, dissolution and related substance in development laboratories etc.

$>$ Prior starting the validation the satisfactory result should be found in mini validation and formulation should be finalized.

Steps involved in Method development are. ${ }^{6,7}$

$>$ Understanding the Physicochemical properties of drug molecule.

Selection of chromatographic conditions.

Developing the approach of analysis.

Sample preparation

Method optimization

Method validation (figure-2)

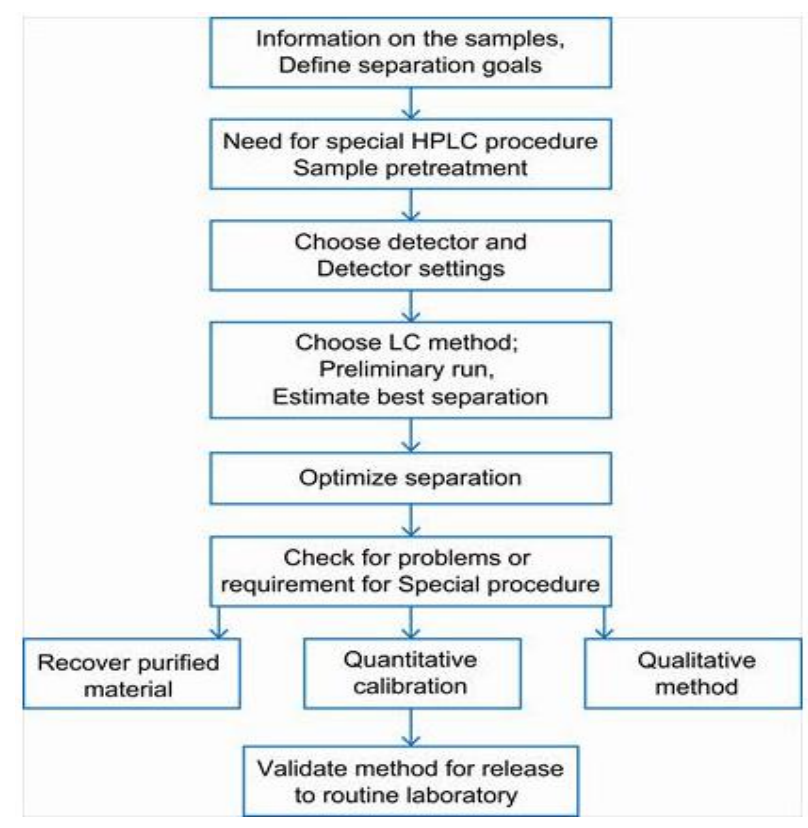

Figure 2: Steps involved in HPLC Method development

2.1 Understanding the physicochemical properties of drug molecules
Physicochemical properties of a drug atom assume an essential role in method development. For Method development one needs to examine the physical properties like dissolvability, Polarity, $\mathrm{pKa}$ and $\mathrm{pH}$ of the drug particle. Polarity is a physical property of a compound. It assists an expert, with deciding the dissolvable and composition of the mobile phase. 6 The dissolvability of atoms can be clarified based on the Polarity of particles. Polar, for example water, and nonpolar, for example benzene, solvents don't blend. By and large, similar to disintegrates like i.e., materials with comparable Polarity are dissolvable in one another. Choice of diluents depends on the dissolvability of analyte. The acidity or basicity of a substance is characterized most normally by the $\mathrm{pH}$ esteem. Choosing an appropriate $\mathrm{pH}$ for ionizableanalytes regularly prompts symmetrical and sharp peak in HPLC.7

\subsection{Selection of chromatographic conditions}

Amid introductory method development, a set of initial conditions (detector, column, mobile phase) is chosen to get the primary "exploring" chromatograms of the sample. Much of the time, these depend on turned reversed-phase partitions on a C18 column with UV identification. A choice on growing either an isocratic or an inclination method ought to be made now.

\subsubsection{Selection of Column:}

A column is obviously, the beginning and focal bit of a chromatograph. A suitably chosen column can create a decent chromatographic division which gives a precise and solid investigation. An inappropriately utilized column can regularly produce perplexity, lacking, and poor partitions which can prompt outcomes that are invalid or complex to interpret.9 The heart of a HPLC system is the column. Changing a column will have the best impact on the goals of analytes amid method development. Picking the best column for application requires thought of stationary phase chemistry, retention capacity, particle size, and column measurementsThe three primary segments of a HPLC column are the equipment, the matrix, and the stationary 
phase. There are a few kinds of matrices for help of the stationary phase, including silica, polymers, alumina, and zirconium. Silica is the most well-known matrix for HPLC column. Silica matrices are powerful, effectively derivatized, produced to predictable circle estimate, and does not will in general pack under strain. Silica is artificially steady to most natural solvents and to low $\mathrm{pH}$ frameworks. One weakness of a silica solid help is that it will dissolve above $\mathrm{pH}$ 7. Lately, silica supported column have been produced for use at high $\mathrm{pH}$. The nature, shape and molecule size of the silica support effect separation. Littler molecule results in a more noteworthy number of hypothetical plates, or expanded. The idea of the stationary phase will decide if a column can be utilized for normal phase or reverse phase chromatography. Normal phase chromatography uses a polar stationary phase and a non-polar mobile phase. For the most part, more polar mixes elute later than non-polar mixes. Regularly utilized invert stage segments and their uses are recorded beneath. Propyl (C3), Butyl (C4), and Pentyl (C5) stages are helpful for particle blending chromatography (C4) and peptides with hydrophobic buildups, and other extensive atoms. C3- C5 columns for the most part hold non-polar solutes all the more ineffectively when contrasted with $\mathrm{C} 8$ or $\mathrm{C} 18$ phases. Models incorporate Zorbax SB-C3, YMC-Pack C4, and Luna C5. These column are commonly less steady to hydrolysis than column with longer alkyl chains. Octyl (C8, MOS) stages have wide pertinence. This stage is less retentive than the C18 stages, yet is still very helpful for pharmaceuticals, nucleosides, and steroids.10Selection of the stationary stage/section is the first and the most critical advance in method development. The improvement of a tough and reproducible technique is incomprehensible without the accessibility of a steady, elite segment. To stay away from issues from irreproducible example maintenance amid technique improvement, it is essential that segments be steady and reproducible. The division selectivity for specific parts shift between the segments of various maker just as between segment creation bunches from a similar producer. Section measurements, silica substrate properties and fortified stationary stage qualities are the primary ones. The utilization of silica-based pressing is supported in a large portion of the present HPLC sections because of a few physical characteristics. 6

\subsubsection{Selection of Chromatographic mode:}

chromatographic modes dependent on the analyte's sub-atomic weight and extremity. All contextual investigations will concentrate on reversed-phase chromatography (RPC), the most widely recognized mode for little natural atoms. Ionizable mixes (acids and bases) are frequently isolated by RPC with supported portable stages (to keep the analytes in a nonionized state) or with ion-pairing reagents. 8

\subsubsection{Optimization of Mobile phase:}

A buffere is an incompletely neutralised acid which opposes changes in $\mathrm{pH}$. Salts, for example, Sodium Citrate or Sodium Lactate are ordinarily used to halfway neutralise the acid. Buffering Capacity is the capacity of the support to oppose changes in $\mathrm{pH}$

I)Buffering Capacity increments as the molar focus (molarity) of the buffer salt/acid solution increases. The closer the buffered $\mathrm{pH}$ is to the pKa, the more noteworthy the Buffering Capacity.

II)Buffering Capacity is communicated as the molarity of Sodium Hydroxide required to build $\mathrm{pH}$ by 1.0. Thought of the effect of $\mathrm{pH}$ on analyte maintenance, kind of buffer to utilize, and its fixation, dissolvability in the natural modifier and its effect on recognition are imperative in turned reversed-phase chromatography (RPC) technique improvement of ionic analytes. An ill-advised decision of support, as far as buffering species, ionic strength and $\mathrm{pH}$, can result in poor or irreproducible maintenance and following reverse-phase partition of polar and ionizable compounds 12,13 ..

\section{Buffer selection}

Decision of buffer is ordinarily administered by the ideal $\mathrm{pH}$. The average $\mathrm{pH}$ extend for turned 
reverse phase on silica-based pressing is $\mathrm{pH} 2$ to 8 . It is important that buffer has a pKa near the ideal $\mathrm{pH}$ since buffer controls $\mathrm{pH}$ best at their pKa. A standard is to pick a buffer with a pKa esteem $<2$ units of the ideal mobile phase $\mathrm{pH}$ (see Table).

\section{General considerations during buffer selection:}

$>$ Phosphate is progressively soluble in methanol/water than in acetonitrile/water or THF/water.

$>$ Some salt buffers are hygroscopic. This may prompt changes in the chromatography (expanded following of fundamental mixes, and perhaps selectivity contrasts).

$>$ Ammonium salts are commonly increasingly dissolvable in natural/water mobile phase $\bullet$

$>$ TFA can debase with time, is unstable, retains at low UV wavelengths.

$>$ Microbial development can rapidly happen in cushioned versatile stages that contain pretty much nothing or noorganic modifier. This development will amass on segment bays and can harm chromatographic performance.

$>$ At $\mathrm{pH}$ more noteworthy than 7, phosphate buffers quickens the disintegration of silica and seriously abbreviates the lifetime of silicabased HPLC segments. On the off chance that conceivable, natural cradles ought to be utilized at $\mathrm{pH}$ more noteworthy than 7 .

> Ammonium bicarbonate buffers as a rule are inclined to $\mathrm{pH}$ changes and are typically steady for just 24 to 48 hours. The $\mathrm{pH}$ of this versatile stage will in general turn out to be progressively essential because of the arrival of carbon dioxide.

$>$ After buffers are readied, they ought to be separated through a 0.2- $\mu \mathrm{m}$ channel.

$>$ Mobile phase ought to be degassed.

\section{Buffer concentration:}

Generally, a bufferconcentration of $10-50 \mathrm{mM}$ is adequate for small molecules. Generally, no more than $50 \%$ organic should be used with a buffer. This will depend on the specific buffer as well as its concentration. Phosphoric acid and its sodium or potassium salts are the most common buffer systems for reversed-phase
HPLC. Phosphonate buffers can be replaced With sulfonate buffers when analyzing organophosphate compounds ${ }^{14,15}$.

\section{Mobile phase}

\section{Mobile Phase Reservoirs}

$>$ Inert container with inert lines leading to the pump are required.

$>$ Reservoir filters $(2-10 \mathrm{~mm})$ at reservoir end of solvent delivery lines

\section{Degassed solvent}

- Vacuum filtration

- $\quad$ Sparge with inert gas $\left(\mathrm{N}_{2}\right.$ or $\left.\mathrm{He}\right)$

- Ultrasonic under vacuum

\section{Isocratic elution:}

A separation that employs a single solvent or solvent mixture of constant composition.

\section{Gradient elution:}

Here at least two solvent systemes that vary essentially in extremity are utilized. After elution is started; the proportion of the solvents is differed in a modified manner, some of the time persistently and now and again in a progression of steps. Partition proficiency is enormously upgraded by gradient elution.

The mobile phase impacts resolution, selectivity and efficiency. In reverse phase chromatography, the mobile phase comprises of a fluid cradle and a non-UV dynamic water miscible natural dissolvable. The impact of the organic and fluid phase and the extents in which they are blended will influence the investigation of the medication atom. Choice of the versatile stage and inclination conditions is subject to the ionogenic idea of the analyte and the hydrophobicity of the analytes in the blend individually. The aqueous buffer fills a few needs. At low $\mathrm{pH}$, the mobile phase protonates free silanols on the column and reduces peak tailing. At adequately low $\mathrm{pH}$ essential analytes are protonated; when ionized the analyte will elute all the more rapidly yet with improved pinnacle shape. Acidic analytes in buffer of adequately low $\mathrm{pH}$ will stay uncharged, expanding maintenance. Then again, at higher 
$\mathrm{pH}$ unbiased essential mixes will be increasingly held, and ionized acidic mixes will elute prior. peak part might be watched if the pKa of a compound is like the pKa of the buffer, and the analyte elutes as both a charged and uncharged animal categories. The $\mathrm{pH}$ of a buffer won't significantly influence the maintenance of non-ionizable sample column.

\subsubsection{Selection of detector and wavelength:}

\section{Selection of detector}

Detector is an imperative part of HPLC. Determination of detector relies upon the substance idea of analytes, potential impedance, point of confinement of discovery required, accessibility and additionally cost of locator. UV-Visible indicator is flexible, double wavelength absorbance detector for HPLC. This finder offers the high affectability required for routine UV-based applications to low-level debasement ID and quantitative investigation. Photodiode Array (PDA) Detector offers progressed optical discovery for Waters investigative HPLC, preparative HPLC, or LC/MS systeme arrangements.Its incorporated programming and optics developments convey high chromatographic and spectral sensitivity. Refractive Index (RI) Detector offers high sensitivity, strength and reproducibility, which make this detector the perfect solution for investigation of parts with restricted or no UV absorption. Multi-Wavelength Fluorescence Detector offers high affectability and selectivity fluorescence location for quantitating low centralizations of target compounds 16 .

\subsection{Developing the approach for analysis:}

While building up the analytical method on RPHPLC the initial step which is pursued, the determinations of different chromatographic parameters like choice of mobile phase, choice of column, choice of flow rate of mobile phase, choice of $\mathrm{pH}$ of mobile phase. These parameters are chosen based on preliminaries and pursued by considering the system suitability parameters. typical parameters of system appropriateness are for example retention time ought to be in excess of $5 \mathrm{~min}$, the hypothetical plates ought to be more than
2000 , the tailling element ought to be under 2 , resolution between 2 peaks ought to be more than 5, \% R.S.D. of the zone of analyte peak in standard chromatograms ought not be more than $2.0 \%$.like other. detection wavelength is normally isobestic point on account of concurrent estimation of 2 components.6components.

\subsection{Sample preparation:}

sample preparation is a basic advance of method development that the examiner must explore. For instance, the expert ought to examine if centrifugation (determine the optimal rpm and time) shaking as well as filtration of the example is required, particularly if there are insoluble segments in the example. The goal is to show that the example filtration does not influence the systematic outcome because of adsorption as well as extraction of leachable. The adequacy of the syringe channels is to a great extent

Dictated by their capacity to expel contaminants/insoluble parts without draining unwanted curios (i.e., extractable) into the filtrate. The sample preparation prosedure ought to be sufficiently portrayed in the separate analytical technique that is connected to a genuine in-process sample or a dosage form for ensuing HPLC examination. The analytical methodology must indicate the producer, kind of channel, and pore size of the channel media. 17 The reason for test readiness is to make a handled example that prompts better investigative outcomes contrasted and the underlying example. The readied test ought to be an aliquot generally free of impedances that is good with the HPLC technique and that won't harm the column.18,20.

\subsection{MethodOptimization:}

The greater part of the optimization of HPLC method development has been centered around the improvement of HPLC conditions.19The mobile phase and stationary phase sytheses should be considered. Improvement of mobile phase parameters is constantly viewed as first as this is a lot simpler 
and advantageous than stationary phase development. To limit the quantity of preliminary chromatograms included, just the parameters that are probably going to significantly affect selectivity in the advancement must be analyzed. Essential control factors in the streamlining of liquid chromatography (LC) method are the distinctive parts of the mobile phase deciding sharpness, dissolvable, slope, flow rate, temperature, test sums, injection volume, and diluents dissolvable sort. This is utilized to detect the ideal harmony among goals and investigation time after agreeable selectivity has been accomplished. The parameters included incorporate segment measurements, segment pressing molecule size and stream rate. These parameters might be changed without influencing limit factor or selectivity.10

\section{ANALYTICAL METHOD VALIDATION}

Method validation such as the process of provided (through scientific studies) that an analytical method is suitable for its projectedusage. Method validationdelivers the method development really specific, Linear, Precise, accurate \& sensitive. The required validation parameters, also called analytical performance characteristics, depend upon the type of analytical method. Allowing to $\mathrm{ICH}$ guideline the validation of analytical methods are outlinebelow.

Method validation is the process to confirm that the analytical procedure employed for a specific test is suitable for its intended use. Analytical method validation is the process of demonstrating that an analytical method is reliable and adequate for its intended purpose. Any method that is utilized to determine results during drug substance and formulation development will have to be validated

\subsection{Parameters of Analytical Method Validation}

Analytical methods have been validated in pursuance of $\mathrm{ICH}$ guidelines of Q2 (R1) ${ }^{[26]}$. Validation parameters are:

1. System suitability
2. Specificity

3. Linearity

4. Precision

5. Accuracy

6. LOD

7. $\mathrm{LOQ}$

8. Robustness

\subsection{System Suitability}

System suitability testing initially accepted by the industry of pharmaceuticals to choose whether a chromatographic system is being used day today in a normal way in pharmaceutical research centers where nature of results is most critical which is reasonable for an unmistakable analysis.

The parameters used in the system suitability tests (SST) report are as follows:

1. Number of theoretical plates or Efficiency (N).

2. Capacity factor (K).

3. Separation or Relative retention $(\alpha)$.

4. Resolution (Rs).

5. Tailing factor $(T)$.

6. Relative Standard Deviation (RSD).

\section{Number of theoretical plates/Efficiency (N)}

In a predefined column, efficiency is characterized as the estimation of the level of peak scattering and it ought to have the column characteristics. The productivity is passed on regarding number of hypothetical plates". The equation of estimation of $N$ is shown cry in the following Figure 3. (Half height method).

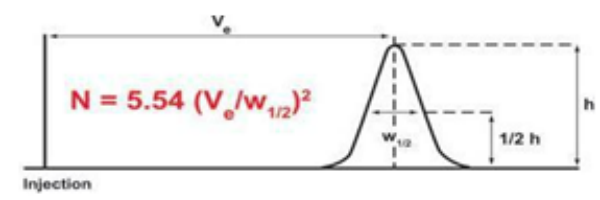

Figure 3. Half height method relating to determination of $\mathrm{N}$.

$\mathrm{N}$ = Efficiency / Number of theoretical plates. 
$\mathrm{V}_{\mathrm{e}}=$ Retention time of analyte.

$\mathrm{h}=$ Height of the peak.

$W_{1 / 2}=$ Gaussian function of the peak width at the half- height.

\section{Sigma/tangential method (USP method)}

With the help of sigma/tangential method $\mathrm{N}$ is calculated which is shown in the following figure 4.Duly noting the formula for calculation of $\mathrm{N}$.

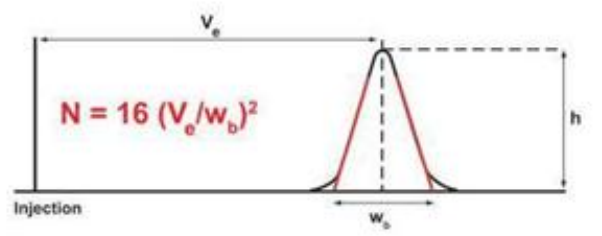

Figure 4.Sigma/tangential method relating to determination of $\mathrm{N}$.

$\mathrm{N}=$ Number of theoretical plates.

$\mathrm{V}_{\mathrm{e}}=$ elution volume, retention time or retention distance $(\mathrm{mL}, \mathrm{sec}$, or $\mathrm{cm})$.

$\mathrm{h}=$ peak height.

$\mathrm{w}_{\mathrm{b}}=$ width of the peak at the base line $(\mathrm{mL}$, $\mathrm{sec}$, or $\mathrm{cm}$ ).

The plate number depends on column length. Theoretical plate number is the amount of column efficiency. Aspecified by plate theory, the analyte will be in immediate equilibrium with stationary phase and column has to be separatedto number of hypothetical plates and every plate involves of a fixed height and analyte spends finite time in the plate. Height equivalent to theoretical plate (HETP) is given by following formula:

$\mathrm{N}=$ Number of theoretical plates.

$V_{e}=$ elution volume, retention time or retention distance $(\mathrm{mL}, \mathrm{sec}$, or $\mathrm{cm})$.

$\mathrm{h}=$ peak height.

$\mathrm{w}_{\mathrm{b}}=$ width of the peak at the base line $(\mathrm{mL}$, $\mathrm{sec}$, or $\mathrm{cm}$ ).

The plate number depends on column length. Theoretical plate number is the amount of column efficiency. Aspecified by plate theory, the analyte will be in immediate equilibrium with stationary phase and column has to be separatedto number of hypothetical plates and every plate involves of a fixed height and analyte spends finite time in the plate. Height equivalent to theoretical plate (HETP) is given by following formula:

HETP $=\mathrm{L} / \mathrm{N}$, Where, (1)

$L=$ length of column.

$\mathrm{N}=$ plate number.

\section{Capacity ratio or Capacity factor $\left(k^{\prime}\right)$}

$$
\mathrm{k}^{\prime}=\frac{t_{\mathrm{R}}-\mathrm{t}_{\mathrm{M}}}{\mathrm{t}_{\mathrm{O}}}
$$

The overhead thought capacity factor occasionally is called as a retention factor which has no dimension and self-governing from flow rate of mobile phase as well as column dimensions which is the measure of extent of retention relating to an analyte relative to an un-retained peak. Where $t_{R}$ implies retention time of the sample peak and retention time of an un-retained peak is $t_{M}$.

$k^{\prime}=0$ means no compound is left in the column. Generally the value of $k^{\prime}$ is $>2$.

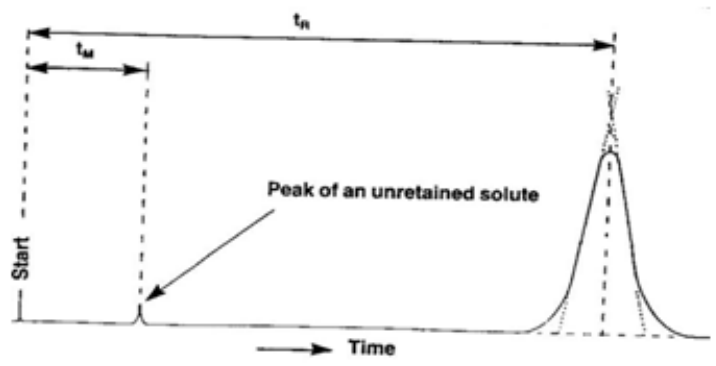

Figure 5. Determination of capacity factor/ capacity ratio.

\section{Relative retention or separation factor $(\alpha)$}

$\alpha=$ Relative retention.

$t_{a} t_{2}$ Retention time calculated from point of injection.

$=$ Unretained peak time (Retention time $\left(t_{R}\right)$ of an inert component not retained by the column). 
$t_{1}=$ the retention time from the point of injection of reference peak defined. (Suppose no reference peak is found, value would be zero).

\section{Resolution (Rs)}

resolution is the capacity of the column to isolate 2 drug in 2 singular peak or chromatographic zones and it is improved by upgrading column length, decrease of molecule size and rising temperature, adjusting the eluent or stationary stage. It very well may be told as far as proportion of ratio of the seperation of two peak by the digressive width normal of the pinnacles. By utilizing the accompanying equation resolution is calculated.

$$
R_{s}=\left(t_{R_{2}}-t_{R_{1}}\right) / 0.5\left(t_{w_{1}}+t_{w_{2}}\right)
$$

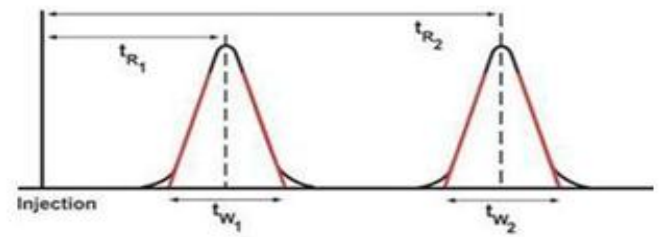

Figure 6: Determination of resolution between two peaks.

$t_{R 1}$ and $t_{R 2}$ are the retention times for the two peaks of components.

$t_{w 1}$ and $t_{w 2}=$ At the baseline lies between tangents drawn to the sides of the peaks. (Tangents are drawn at 0.6 times the peak height). If the peaks are correctly symmetric, provided the valley between the two peaks should touch the baseline Rs is 1.5. Generally good value of resolution is Rs $\geq 2$ should be adequate and preferred normally.

\section{Resolution factor (R)}

Resolution is a function of capacity factor, function of selectivity and a function of efficiency (or) number of theoretical plates (N). In order to separate any two peaks you must have right capacity factor ideally between 2 and 10 , but appropriate selectivity is required i.e., ideally 1.2 and enough efficiency i.e., number of theoretical plates (more than 2000 theoretical plates). Resolution should be $\geq 1.5$. 1.5 defines baseline resolution.

$$
\mathrm{R}=\frac{\mathrm{k}^{\prime}}{1+\mathrm{k}^{\prime}} \times \frac{\alpha-1}{\alpha} \times \sqrt{\frac{\mathrm{N}}{4}}
$$

\section{Tailing factor or Asymmetry factor}

Chromatographic peak accepted to have a Gaussian shape under perfect conditions. Anyway in down to earth conditions, there is dependably a deviation from typical dispersion which shows non-uniform movement and nonuniform conveyance process. Consequently the administrative associations like USP and EP have suggested this as one of the framework reasonableness parameter. The asymmetry factor and following element are generally same and seldom precise and measure up to as a rule. Qualities ought to typically between 1.01.5 and values more noteworthy than 2 are inadmissible. The pinnacle asymmetry is registered by using the accompanying recipe.

$A_{s}=B / A$

Where:

$A_{s}=$ peak asymmetry factor .

$B=$ distance from the point at peak midpoint to the trailing edge.

(measured at $10 \%$ of peak height).

$A=$ distance from the leading edge of peak to the midpoint.

(measured at $10 \%$ of peak height).

Ideally, peaks should be Gaussian in shape or totally symmetrical. Determination of tailing and asymmetric factor is shown in Figure 7.
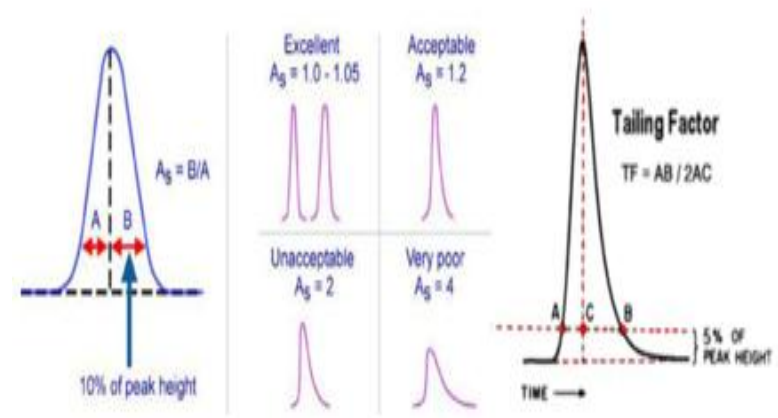

Figure 7: Determination of tailing and asymmetric factor. 
Acceptance criteria (limits) of system suitability parameters are shown in the following Table 1.

Table 1: Acceptance criteria for system suitability parameters.

\begin{tabular}{|c|c|c|}
\hline Sr. No & Parameter name & $\begin{array}{l}\text { Acceptance } \\
\text { criteria }\end{array}$ \\
\hline 1 & $\begin{array}{l}\text { Number of theoretical } \\
\text { plates or Efficiency }(\mathrm{N})\end{array}$ & $>2000$ \\
\hline 2 & Capacity factor (K) & $<1$ \\
\hline 3 & $\begin{array}{l}\text { Separation or Relative } \\
\text { retention }(\alpha)\end{array}$ & $>1$ \\
\hline 4 & Resolution (Rs) & $>1.5$ \\
\hline 5 & $\begin{array}{c}\text { Tailing factor or } \\
\text { Asymmetry }(T)\end{array}$ & $<2$ \\
\hline 6 & $\begin{array}{l}\text { Relative Standard } \\
\text { Deviation (RSD) }\end{array}$ & $<2$ \\
\hline
\end{tabular}

\subsection{Specificity}

One of the noteworthy features of HPLC is its capacity to produce signals free from interference. Specificity alludes to the capacity of the analytical method to separate and evaluate the analyte in complex blends. An investigation of specificity is to be directed amid the assurance of contaminations and validation of identification proof tests.

An $\mathrm{ICH}$ rule guideline specificity as capacity to evaluate unequivocally the analyte within the sight of different compounds that might probably be available. Regularly these may be contaminations, degradants, matrix, and so on. The definition has the accompanying ramifications:

Identification test: Identification tests ought to have the capacity to separate mixes of intently related structure which are relied upon to be available i.e., to guarantee character of an analyte.

$>$ Purity test: To guarantee that the scientific system performed permits a precise proclamation of content of the pollution of an analyte for example related substances, remaining solvents content, substantial metals, and so on

Assay: To arrive at a precise outcome, this allows a right report on the strength or substance of analytein an sample.

\subsection{Linearity and Range}

The linearity of a method is a proportion of how well an calibration plot of response versus concentration approximates a straight line. Linearity can be surveyed by performing single estimations at a few analyte concentration. The information is then handled utilizing a direct least-squares relapse. The subsequent plot slant, capture and relationship coefficient give the ideal data on linearity.

\subsection{Precision}

The precision of an analytical method speaks to the closeness of understanding between a progression of precision got from numerous testing of the equivalent homogenous sample under the comparative explanatory conditions and it is partitioned into 3 classifications.

$>$ Repeatability: precision under same operating conditions, same analyst over a short period of time.

$>$ Intermediate precision: method is tested on multiple days, instruments, analysts etc.

Reproducibility: inter-laboratory studies.

The ICH guidelines recommend that repeatability should be conformed properly employ at least 9 determinations with specified range for the procedur or a minimum of 6 determinations at $100 \%$ of the test concentration.

\subsection{Accuracy}

The accuracy of a measurement is defined as the closeness of the measured value to the true value. In a method with high accuracy, a sample (whose "true value" is known) is analyzed and the measured value is identical to the true value. Typically, accuracy is represented and determined by recovery 
studies. There are three ways to determine accuracy:

1. Comparison to a reference standard.

2. Recovery of the analyte spiked into blank matrix.

3. Standard addition of the analyte.

It should be clear how the individual or total impurities are to be determined.

\subsection{Limit of detection}

Limit Of Quantification is intent by the analysis of samples with studied concentration of analyze and by initiate that minimum level at which the analyte can reliably detected, but not required quantities as precise value, under the express experimental conditions. The detection limit is generally expressed in the concentration of analyte (ppm) in the sample.

A number of approaches are recommended by the $\mathrm{ICH}$ for determining the detection limit of sample, depending on instrument used for analysis, nature of analyte and suitability of the method. The acceptable approaches are

1. Visual evaluation.

2. Signal-to-noise ratio.

3. Standard deviation of the response.

4. Standard deviation of the slope of linearity plot.

The formula for calculating LOD is

$\mathrm{LOD}=3.3 \delta / \mathrm{S}(7)$

Where $\delta=$ standard deviation of intercepts of calibration curves.

$S=$ the slope of linearity plot

\subsection{Limit of quantitation}

Limit of quantization is the minimum concentration of drug in a sample which is determined with suitable precision and accuracy under the declared experimental conditions. Similar to LOD, ICH suggest the given four methods for approximation of LOQ. The acceptable approaches are

1. Visual evaluation.

2. Signal-to-noise ratio.
3. Standard deviation of the response.

4. Standard deviation of the slope of linearity plot.

The formula for calculating LOQ is

$\mathrm{LOQ}=10 \delta / \mathrm{S}(8)$

Where $\delta=$ standard deviation of response.

$S=$ Mean of slopes of the calibration curves.

\subsection{Robustness}

Robustness is defined by the quantum of the capability of an analytical method to stay without changed by mini deliberate changes in method parameters. The changeable method parameters in HPLC technique may intertwine flow rate, column temperature, sample temperature, mobile phase and $\mathrm{Ph}$ composition.

\section{Conclusion}

In years current development of the analytical methods for recognition, clarity evaluation and quantification of drugs has received a great deal of notice in the field of pharmaceutical analysis. This review report HPLC method development and validation in simple way. A common and very simple advance toward for the HPLC method development for the dissociation of compounds was discussed. Knowledge of the physiochemical properties of the primary compound is of importance preceding to the any HPLC method development.

A selection of buffer and mobile phase composition plays a substantial role on the separation selectivity. Final modification can be performed by changing the gradient slope, temperature and flow rate as well as the type and concentration of mobile-phase moderate. Optimized method is validated with various parameters (e.g. specificity, precision, accuracy, detection limit, linearity, etc.) as per $\mathrm{ICH}$ guidelines.

\section{Abbreviations}

HPLC High Performance Liquid Chromatography 
$\mathrm{ICH}$ International conference on Harmonization

$\begin{array}{ll}\text { Id } & \text { Internal Diameter } \\ \text { LC } & \text { Liquid Chromatography } \\ \text { LOD } & \text { Limit of Detection } \\ \text { LOQ } & \text { Limit of Quantitation } \\ \text { MmMili meter } \\ \text { MS } & \text { Mass Spectrometry } \\ \text { ODS } & \text { Octyldecylsilane } \\ \text { RI } & \text { Refractive index } \\ \text { THF } & \text { Tetrahydrofuran } \\ \text { USP } & \text { United states Pharmacopeia } \\ \text { Hm } & \text { Micron }\end{array}$

\section{References:}

1. V. Gupta, A.D. K. Jain, N.S. Gill, K. Gupta, Development and validation of HPLC method - a review, Int. Res J Pharm. App Sci., 2(4) (2012) 17-25

2. Y. Kazakevich, R. Lobrutto, HPLC for Pharmaceutical Scientists, John Wiley \& Sons, New Jersey, 2007.

3. Ahuja, H. Rasmussen, Development for Pharmaceuticals, Vol.8 Separation Science and Technology, Elsevier, New York 2007

4. M.S. Azim, M. Mitra, P.S. Bhasin, HPLC method development and validation: $A$ review, Int. Res. J. Pharm. 4(4) (2013) 3946.

5. B.V. Rao, G.N. Sowjanya1, A. Ajitha, V.U.M. Rao, Review on stability indicating hplc method development, World Journal of Pharmacy and Pharmaceutical Sciences, 4(8) (2015) 405-423.

6. M.S. Charde, A.S. Welankiwar, J. Kumar, Method development by liquid chromatography with validation, International Journal of Pharmaceutical Chemistry, 04 (02)(2014) 57-61.

7. S. Sood, R. Bala, N.S. Gill, Method development and validation using HPLC technique - A review, Journal of Drug Discovery and Therapeutics 2 (22) 2014, 18-24.

8. M.W. Dong, Modern Hplc for practicing scientists, John Wiley \& Sons, New Jersey, 2006.
9. P.K. Singh, M. Pande, L.K. Singh, R.B. Tripathi, steps to be considered during method development and validation for analysis of residual solvents by gas chromatography, Int. Res J Pharm. App Sci., 3(5) (2013) 74-80.

10. B. Prathap, G.H.S. Rao, G. Devdass, A. Dey, N. Harikrishnan, Review on Stability Indicating HPLC Method Development, International Journal of Innovative Pharmaceutical Research.3(3) (2012) 229237.

11. B. Sriguru, N.P. Nandha, A.S.Vairale, A.V. Sherikar, V. Nalamothu, Development and validation of stability indicating HPLC method for the estimation of 5Fluorouracil and related substances in topical formulation, Int. J. Res. Pharm. Sci. 1 (2) (2010) 78-85.

12. C.K. Kaushal, B. Srivastava, A process of method development: A chromatographic approach, J. Chem. Pharm. Res. 2(2) (2010) 519-545.

13. N.Toomula, A. Kumar, S.D.Kumar, V.S. Bheemidi, Development and Validation of Analytical Methods for Pharmaceuticals, J Anal Bioanal Techniques. 2(5) (2011) 1-4.

14. K. Kardani, N. Gurav, B. Solanki, P. Patel, B. Patel, RP-HPLC Method Development and Validation of Gallic acid inPolyherbal Tablet Formulation, Journal of Applied Pharmaceutical Science. 3 (5) (2013) 37-42.

15. B. Nigovic, A. Mornar, M. Sertic, Chromatography - The Most Versatile Method of Chemical Analysis, Intech (2012) 385-425.

16. G. P. Carr and J. C. Wahlichs.„,A practical approach to method validation in pharmaceutical analysis", J. Pharm, Biomed. Anal,8,1990,613-618.

17. United States Pharmacopoeia, 24 , National Formulary 19, section <1225> "Validation of compendial methods". US Pharmacopoeial convention, Rockville, Validation of analytical procedures text and methodology Q2 (R1), November, 2000: 2005 